Elsevier required licence: (C) 2018

This manuscript version is made available under the CC-BY-NC-ND 4.0 license http://creativecommons.org/licenses/by-nc-nd/4.0/

The definitive publisher version is available online at https://doi.org/10.1016/j.automatica.2018.08.009 


\title{
Comparison of Two Different Objective Functions in 2D Point Feature SLAM
}

\author{
Heng Wang ${ }^{\mathrm{a}, \mathrm{b}}$, Shoudong Huang ${ }^{\mathrm{c}}$, Guanghong Yang ${ }^{\mathrm{b}}$, Gamini Dissanayake ${ }^{\mathrm{c}}$ \\ ${ }^{\text {a }}$ School of Automation, University of Science and Technology Beijing, Beijing, 100083, P. R. China. \\ ${ }^{\mathrm{b}}$ College of Information Science and Engineering, Northeastern University, Shenyang, 110819, P. R. China. \\ ${ }^{\mathrm{c}}$ Centre for Autonomous Systems, Faculty of Engineering and Information Technology, University of Technology, Sydney, Australia.
}

\begin{abstract}
This paper compares two different objective functions in 2D point feature Simultaneous Localization and Mapping (SLAM). It is shown that the objective function can have a significant impact on the convergence of the iterative optimization techniques used in SLAM. When Frobenius norm is adopted for the error term of the orientation part of odometry, the SLAM problem has much better convergence properties, as compared with that using the angle difference as the error term. For one-step case, we have proved that there is one and only one minimum to the SLAM problem, and strong duality always holds. For two-step case, strong duality always holds except when three very special conditions hold simultaneously (which happens with probability zero), thus the global optimal solution to primal SLAM problem can be obtained by solving the corresponding Lagrangian dual problem in most cases. Further, for arbitrary m-step cases, we also show using examples that much better convergence results can be obtained. Simulation examples are given to demonstrate the different convergence properties using two different objective functions.
\end{abstract}

Key words: 2D Point Feature SLAM; dimension reduction; convergence properties; strong duality.

\section{Introduction}

The main task of Simultaneous Localization and Mapping (SLAM) is to estimate robot poses and environment features simultaneously. SLAM plays an important role in robot navigation in environments where GPS signals are seriously sheltered, such as indoor, underwater, underground environments etc (Cadena, Carlone, Carrillo, Latif, Scaramuzza, Neira, Reid \& Leonard 2016). In the past decades, many efficient algorithms and methods for SLAM have been proposed. In order to obtain a globally consistent representation of environment and optimal estimation of robot poses, graph theory, convex optimization theory and probability theory have been widely used in the literature. Note that

\footnotetext{
ऋ This work has been supported in part by the Funds of National Science of China (Grant No. 61673098), and the Fundamental Research Funds for the Central Universities. This paper was not presented at any IFAC meeting. Corresponding author H. Wang. Tel. +86-02483681939. Fax +86-02483681939.

Email addresses: hengwang@ustb.edu. cn (Heng Wang), Shoudong. Huangeuts . edu.au (Shoudong Huang), yangguanghongdise.neu.edu.cn (Guanghong Yang), Gamini.Dissanayakeduts.edu.au (Gamini Dissanayake).
}

one important part of a SLAM system is called back-end which aims to use the odometry and observation information to produce the maximum a posteriori estimate, the standard formulation origins from (Lu \& Milios 1997)(Gutmann \& Konolige 1999) and followed by many papers (Thrun \& Montemerlo 2006)(Dellaert \& Kaess 2006)(Grisetti, Stachniss, Grzonka \& Burgard 2007)(Kaess, Johannsson, Roberts, Ila, Leonard \& Dellaert 2012).

SLAM is a non-convex nonlinear optimization problem, and it is supposed to have many local minima, however, recent researches have shown some interesting phenomena, for example, it seems that iterative algorithms are more apt to converge to globally optimal solution, even when starting with very poor quality initial values (Olson, Leonard \& Teller 2006)(Grisetti, Stachniss \& Burgard 2009)(Huang, Lai, Frese \& Dissanayake 2010). For one-step SLAM problem where robot only moves one step, the nonlinear structure has been analyzed in (Wang, Huang, Frese \& Dissanayake 2013), necessary and sufficient conditions guaranteeing globally optimal solution have been proved (Wang et al. 2013)(Wang, Hu, Huang \& Dissanayake 2012), and it is also proved that there are at most two minima for any onestep SLAM optimization problem. In (Carlone 2013), basin of convergence for Gauss-Newton method is estimated; and 
in (Carlone, Calafiore, Tommolillo \& Dellaert 2016), it is proved that under strong duality condition, global optimal solution to pose graph SLAM can be obtained through solving a convex semi-definite programming (SDP) problem. Further, through using Lagrangian duality of SLAM problem, some verification algorithms have been proposed, which is applied to judge whether a given SLAM solution is optimal or not (Carlone \& Dellaert 2015)(Carlone et al. 2016). On the other hand, by investigating the special structure of SLAM problem, some convex relaxation methods have been presented in (Liu, Huang, Dissanayake \& H.Wang 2012)(Rosen, DuHadway \& Leonard 2015) which avoid converging to local minima, and in (Khosoussi, Huang \& Dissanayake 2015), nonlinear separability between translation and rotation is well investigated such that computation speed is improved greatly, while in (Carlone, Aragues, Castellanos \& Bona 2014), a linear approximation method named LAGO (Linear Approximation for pose Graph Optimization) is proposed which can reduce the risk of being trapped in a local minimum.

It should be noted that there are two different kinds of objective functions used in the above mentioned 2D SLAM formulations. One is using $\operatorname{wrap}(\cdot)$ function to constrain the error term relating to the orientation of odometry into $(-\pi, \pi]$ interval which is commonly referred to as angular or geodesic distance (Carlone, Tron, Daniilidis \& Dellaert 2015). Another is using Frobenius norm to deal with orientation part of odometry. Particularly, in (Carlone et al. 2015)(Hartley, Trumpf, Dai \& Li 2013), a comprehensive description of these objective functions and rotation estimation are presented, and in (Carlone et al. 2015), the authors attempt to relate these two objective functions firstly up to the first order. This paper extends the investigation of nonlinear structure analysis as proposed in (Wang et al. 2013) and (Wang, Huang, Khosoussi, Frese, Dissanayake \& Liu 2015) by modifying the objective function using Frobenius norms which was proposed in (Carlone et al. 2016)(Rosen et al. 2015)(Rosen, Carlone, Bandeira \& Leonard 2016). The main contribution of this paper is twofold. Firstly, for one-step SLAM problem, we show that strong duality between primal SLAM and corresponding convex programming problem always holds, and for two-step SLAM problem, we present clear conditions for this strong duality which are expressed using observation and odometry data. Secondly, we compare the convergence to global optimal solution for both new objective function (using Frobenius norm to deal with orientation part of odometry) and former objective function (using a $w r a p(\cdot)$ function to constrain the error term of the orientation part of odometry directly); the comparison results show that using new objective function formulation results in much better convergence results.

Notations: Throughout the paper, $\otimes$ denotes the Kronecker product, superscript $T$ and -1 stand for, respectively, the transposition and the inverse of a matrix; $C \succ D$ means that matrix $C-D$ is positive definite; $I$ and $I_{n}$ denote the identity matrix with compatible dimension and dimension $n$; 0 represents the zero matrix with compatible dimension, and $\|\mathbf{e}\|_{C}^{2}=\mathbf{e}^{T} C \mathbf{e}$, where $C \succ 0$ and $\mathbf{e}$ is a vector. $\operatorname{wrap}(\cdot)$ is the function that maps an angle to its equivalent angle in $(-\pi, \pi]$. The symbol $\operatorname{diag}\left\{C_{1}, \ldots, C_{n}\right\}$ denotes a blockdiagonal matrix whose diagonal blocks are $C_{1}, \ldots, C_{n}$.

\section{Outline of the paper}

In order to make the paper more accessible, an outline of the remaining sections is provided before the main body of the paper as follows:

- In Section 3, two kinds of objective functions, the old one using $\operatorname{wrap}(\cdot)$ and the new one using Frobenius norm, are formulated for 2D point feature SLAM problems;

- In Section 4, the dimension of the primal SLAM problem using the new objective function is reduced, where both two-step and $m$-step cases are considered;

- In Section 5, the primal unconstrained optimization problem in Section 4 is converted into a constrained optimization problem, which is essential for later solution analysis in Sections 6 and 7;

- In Section 6, the global optimal solution of one-step SLAM problem is analyzed by investigating the strong duality between primal SLAM problem and its corresponding relaxed convex optimization problem;

- In Section 7, conditions to obtain the global optimal solution of two-step SLAM problem are drawn through investigating the corresponding Lagrangian dual function to the primal SLAM problem;

- In Section 8, some simulation results are provided to show the advantage of using Frobenius norm in objective function for $m$-step case, where the convergence performance is compared with those of the objective functions using $\operatorname{wrap}(\cdot)$ as presented in (Wang et al. 2013)(Wang et al. 2015);

- In Section 9, some conclusions of this paper and future work are provided.

\section{Two objective functions used in SLAM}

In this section, we will introduce two kinds of objective function that have been used in the literature.

Assume that $n$ 2D point features $\left\{f_{k}\right\}_{k=1}^{n}$ are observed from a sequence of $m+12 \mathrm{D}$ robot poses $\left\{r_{i}\right\}_{i=0}^{m}$. We use $\mathbf{z}_{k}^{i}$ to denote the observation made from pose $r_{i}$ to feature $f_{k}$, $\mathbf{o}_{i}(1 \leq i \leq m)$ denote the odometry measurement between pose $r_{i-1}$ and pose $r_{i}$ which includes both the relative translation measurement $\mathbf{o}_{i}^{x y}$ and the relative rotation measurement $o_{i}^{\phi}$. Both the odometry and observations are corrupted by zero-mean Gaussian noises with covariance matrices $P_{z_{k}^{i}}, P_{o_{i}^{x y}}$ and $p_{o_{i}^{\phi}}$, respectively. $\mathbf{x}_{f_{k}}=\left[\begin{array}{ll}x_{f_{k}} & y_{f_{k}}\end{array}\right]^{T}$ denotes the position of feature $f_{k} . \mathbf{x}_{r_{i}}=\left[\begin{array}{ll}x_{r_{i}} & y_{r_{i}}\end{array}\right]^{T}$ denotes the position of robot pose $r_{i}$ while $\phi_{r_{i}}$ denotes the orientation of robot pose $r_{i} . R\left(\phi_{r_{i}}\right)$ is the rotation matrix corresponding 
to $\phi_{r_{i}}$ defined by:

$$
R\left(\phi_{r_{i}}\right) \triangleq\left[\begin{array}{cc}
\cos \phi_{r_{i}} & -\sin \phi_{r_{i}} \\
\sin \phi_{r_{i}} & \cos \phi_{r_{i}}
\end{array}\right]
$$

We first state the definitions of spherical matrices which were defined in (Wang et al. 2013)(Wang et al. 2015).

Definition 1: $A \in \mathbb{R}^{2 \times 2}$ is called spherical if it commutes with $R(\phi)$ (defined in (1)) for every $\phi$. i.e. $A R(\phi)=R(\phi) A$ for every $\phi . B \in \mathbb{R}^{3 \times 3}$ is called spherical if it has the format of $B=\operatorname{diag}\{A, a\}$ where $A \in \mathbb{R}^{2 \times 2}$ is spherical and $a$ is a real number.

Definition 2: Let $\bar{R}_{k}(\phi)$ be the block-diagonal matrix with $k$ blocks where each block is a $2 \times 2$ rotation matrix $R(\phi)$. That is

$$
\bar{R}_{k}(\phi) \triangleq \operatorname{diag}\{R(\phi), \ldots, R(\phi)\}
$$

Matrix $A \in \mathbb{R}^{2 n \times 2 m}$ is called spherical if $A \bar{R}_{m}(\phi)=$ $\bar{R}_{n}(\phi) A$ for every $\phi$.

When covariance matrices $P_{z_{k}^{i}}$ and $P_{o_{i}^{x y}}$ are spherical (Wang et al. 2015)(Carlone et al. 2016)(Rosen et al. 2015), SLAM can be formulated as solving a non-linear least squares (NLLS) problem which is to minimize

$$
\begin{aligned}
F_{0}(\mathbf{x})= & \sum_{i=0}^{m} \sum_{j=1}^{n_{i}}\left\|\left(\mathbf{x}_{f_{k}}-\mathbf{x}_{r_{i}}\right)-R\left(\phi_{r_{i}}\right) \mathbf{z}_{k}^{i}\right\|_{P_{z_{k}^{i}}^{-1}}^{2} \\
& +\sum_{i=1}^{m}\left\|\left(\mathbf{x}_{r_{i}}-\mathbf{x}_{r_{i-1}}\right)-R\left(\phi_{r_{i-1}}\right) \mathbf{o}_{i}^{x y}\right\|_{P_{o_{i}^{x y}}^{-1}}^{2} \\
& +\sum_{i=1}^{m} p_{o_{i}^{\phi}}^{-1}\left(\operatorname{wrap}\left(o_{i}^{\phi}-\phi_{r_{i}}+\phi_{r_{i-1}}\right)\right)^{2}
\end{aligned}
$$

where $n_{i}$ denotes the number of features observed from robot pose $r_{i}$ and $k$ is the global index of the $j$-th feature observed from pose $r_{i}$, and the coordinate frame is defined by the robot pose $r_{0}$, that is, $\mathbf{x}_{r_{0}}=\left[\begin{array}{ll}0 & 0\end{array}\right]^{T}, \phi_{r_{0}}=0$, $R\left(\phi_{r_{0}}\right)=R(0)=I$.

The state vector contains all the robot poses and all the feature positions that are to be estimated.

$$
\mathbf{x} \triangleq\left[\begin{array}{llllllll}
\mathbf{x}_{f_{1}}^{T} & \cdots & \mathbf{x}_{f_{n}}^{T} & \mathbf{x}_{r_{1}}^{T} & \phi_{r_{1}} & \cdots & \mathbf{x}_{r_{m}}^{T} & \phi_{r_{m}}
\end{array}\right]^{T}
$$

In (Carlone et al. 2016)(Rosen et al. 2015), the Frobenius norm is used to deal with the orientation part of odometry in (3), which generate the following objective function

$$
\begin{aligned}
F(\mathbf{x})= & \sum_{i=0}^{m} \sum_{j=1}^{n_{i}}\left\|\left(\mathbf{x}_{f_{k}}-\mathbf{x}_{r_{i}}\right)-R\left(\phi_{r_{i}}\right) \mathbf{z}_{k}^{i}\right\|_{P_{z_{k}^{i}}^{-1}}^{2} \\
& +\sum_{i=1}^{m}\left\|\left(\mathbf{x}_{r_{i}}-\mathbf{x}_{r_{i-1}}\right)-R\left(\phi_{r_{i-1}}\right) \mathbf{o}_{i}^{x y}\right\|_{P_{o_{i}^{x x y}}^{-1}}^{2} \\
& +\sum_{i=1}^{m} \frac{\gamma_{i}}{2}\left\|R\left(o_{i}^{\phi}\right)-R^{T}\left(\phi_{r_{i-1}}\right) R\left(\phi_{r_{i}}\right)\right\|_{F}^{2}
\end{aligned}
$$

where $\gamma_{i}>0$ is a weight, $\|\cdot\|_{F}$ is the Frobenius norm.

In the following sections, we firstly formulate an alternative form for objective function (5) with reduced number of variables, then provide condition for strong duality between primal SLAM problem and its corresponding relaxed convex optimization problem using observation and odometry data, in addition, we compare performance of convergence to globally optimal solution by applying objective function (5) with that by applying objective function (3).

\section{Dimension reduction of $m$-step point feature SLAM}

In this section, we study the dimension reduction of SLAM problem formulation (5). Firstly, two-step SLAM case is considered, then the result is extended to $m$-step case (with $m+1$ robot poses $\left.r_{0}, r_{1}, \cdots, r_{m}\right)$.

\subsection{Two-step SLAM}

When the covariance matrices are spherical, by (5), the objective function of the two-step SLAM problem can be written in a compact form as below (note $\phi_{r_{0}}=0$ ) similar to (Wang et al. 2015)

$$
\begin{aligned}
F(\mathbf{x}) & =\left\|A^{T} \mathbf{x}_{L}-\check{R}\left(\phi_{r_{0}}, \phi_{r_{1}}, \phi_{r_{2}}\right) \mathbf{z}\right\|_{C}^{2} \\
& +\frac{\gamma_{1}}{2}\left\|R\left(o_{1}^{\phi}\right)-R\left(\phi_{r_{1}}\right)\right\|_{F}^{2} \\
& +\frac{\gamma_{2}}{2}\left\|R\left(o_{2}^{\phi}\right)-R^{T}\left(\phi_{r_{1}}\right) R\left(\phi_{r_{2}}\right)\right\|_{F}^{2}
\end{aligned}
$$

where $\mathbf{x}_{L} \triangleq\left[\begin{array}{lllll}\mathbf{x}_{f_{1}}^{T} & \ldots & \mathbf{x}_{f_{n}}^{T} & \mathbf{x}_{r_{1}}^{T} & \mathbf{x}_{r_{2}}^{T}\end{array}\right]^{T}$, and

$$
A \triangleq A^{0} \otimes I_{2}
$$

where $A^{0} \in \mathbb{R}^{(n+2) \times\left(n_{0}+n_{1}+n_{2}+2\right)}$ ( $n$ is the total number of features observed, $n_{i}$ is the number of features observed from pose $\left.r_{i}, i=0,1,2\right)$ is the reduced incidence matrix of the directed graph of the SLAM problem (the direction of each edge is from the robot pose to the observed feature or from the robot pose $i$ to the robot pose $i+1$, as shown in Fig. $1)$. The reduced incidence matrix is obtained by deleting the 
row that corresponds to the "origin" in the incidence matrix of graph $^{1}$.

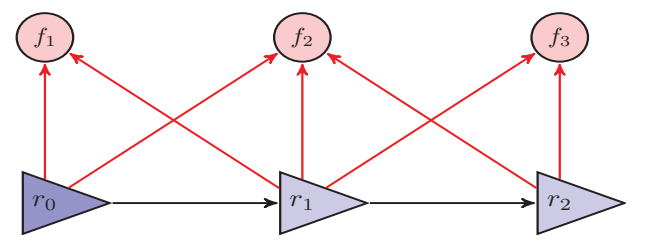

Fig. 1. An example of two-step SLAM with three features.

For example, for the two-step SLAM with three features as shown in Fig. 1 with pose $r_{0}$ as the "origin", the reduced incidence matrix $A^{0}$ can be written as

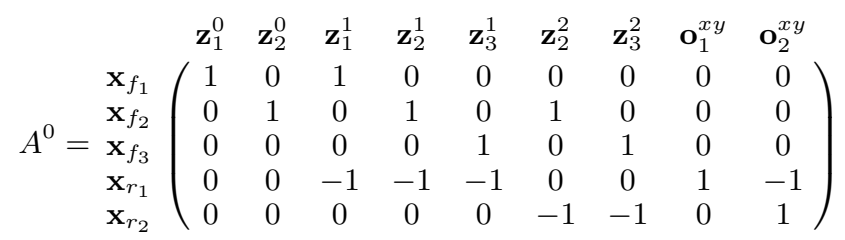

The other matrices/vectors in (6) are (note that $\phi_{r_{0}}=0$ )

$$
\begin{aligned}
& \check{R}\left(\phi_{r_{0}}, \phi_{r_{1}}, \phi_{r_{2}}\right) \\
& \triangleq \operatorname{diag}\left\{\bar{R}_{n_{0}}\left(\phi_{r_{0}}\right), \bar{R}_{n_{1}}\left(\phi_{r_{1}}\right), \bar{R}_{n_{2}}\left(\phi_{r_{2}}\right), R\left(\phi_{r_{0}}\right), R\left(\phi_{r_{1}}\right)\right\} \\
& =\operatorname{diag}\left\{\bar{R}_{n_{0}}(0), \bar{R}_{n_{1}}\left(\phi_{r_{1}}\right), \bar{R}_{n_{2}}\left(\phi_{r_{2}}\right), R(0), R\left(\phi_{r_{1}}\right)\right\} \\
& \mathbf{z} \triangleq\left[\left(\mathbf{z}_{f}^{0}\right)^{T}\left(\mathbf{z}_{f}^{1}\right)^{T}\left(\mathbf{z}_{f}^{2}\right)^{T}\left(\mathbf{o}_{1}^{x y}\right)^{T}\left(\mathbf{o}_{2}^{x y}\right)^{T}\right]^{T} \\
& C \triangleq \operatorname{diag}\left\{C_{z}^{0}, C_{z}^{1}, C_{z}^{2}, C_{p}\right\}
\end{aligned}
$$

where $\bar{R}_{k}(\phi)$ is defined in (2), and

$$
\begin{aligned}
\mathbf{z}_{f}^{i} & =\left[\left(\mathbf{z}_{k_{1}}^{i}\right)^{T} \ldots\left(\mathbf{z}_{k_{n_{i}}}^{i}\right)^{T}\right]^{T}, i=0,1,2 \\
C_{z}^{i} & =\operatorname{diag}\left\{P_{z_{k_{1}}^{i}}^{-1}, \ldots, P_{z_{k_{n_{i}}}^{i}}^{-1}\right\}, i=0,1,2 \\
C_{p} & =\operatorname{diag}\left\{P_{o_{1}^{x y}}^{-1}, P_{o_{2}^{x y}}^{-1}\right\} .
\end{aligned}
$$

According to Definition 1, $A, C$ are both spherical matrices.

Before stating the main results, we introduce more notations. Denote

$$
A_{i} \triangleq A_{i}^{0} \otimes I_{2}, \quad i=0,1,2
$$

where $A_{i}^{0}$ is similar to the reduced incidence matrix $A^{0}$ but assuming only edges originating from pose $r_{i}$ is available. Thus

$$
A^{0}=A_{0}^{0}+A_{1}^{0}+A_{2}^{0}, \quad A=A_{0}+A_{1}+A_{2} .
$$

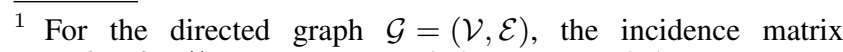
$\mathcal{A} \in\{0,1\}^{n_{v} \times n_{e}}$ where $n_{v}=|\mathcal{V}|$ and $n_{e}=|\mathcal{E}|$. For the $k^{\prime}$ th edge $\left(i_{k}, j_{k}\right) \in \mathcal{E}, \mathcal{A}_{i_{k} k}=-1$ and $\mathcal{A}_{j_{k} k}=1$. The other elements of $\mathcal{A}$ are zero.
See (Wang et al. 2015) for more details on the formulation of $A^{0}$ and $A_{i}^{0}$. Furthermore, we define

$$
Q_{i} \triangleq C A_{i}^{T}\left(A C A^{T}\right)^{-1} A C, \quad i=0,1,2 .
$$

Now the dimension reduction result for two-step SLAM is stated in the following theorem.

Theorem 1: The two-step SLAM problem (minimizing the objective function $F(\mathbf{x})$ in (6)) is equivalent to minimizing the following nonlinear function with two variables

$$
\begin{gathered}
f_{0}\left(\phi_{r_{1}}, \phi_{r_{2}}\right)=c_{1}-2 \sum_{0 \leq i<2} \sum_{i<j \leq 2} b_{i j} \cos \left(\phi_{r_{j}}-\phi_{r_{i}}-\phi_{i j 0}\right) \\
-2 \sum_{i=1}^{2} \gamma_{i} \cos \left(\phi_{r_{i}}-\phi_{r_{i-1}}-o_{i}^{\phi}\right)
\end{gathered}
$$

where $c_{1}=\mathbf{z}^{T} C \mathbf{z}-\sum_{i=0}^{2} b_{i i}+2 \sum_{i=1}^{2} \gamma_{i}$, and for $i, j=$ $0,1,2$,

$$
\begin{aligned}
& b_{i j} \triangleq \sqrt{\left(\mathbf{z}^{T} Q_{i} \mathbf{z}_{j}\right)^{2}+\left(\mathbf{z}^{T} Q_{i} \mathbf{z}_{j}^{\perp}\right)^{2}} \\
& \phi_{i j 0} \triangleq \operatorname{atan} 2\left(\mathbf{z}^{T} Q_{i} \mathbf{z}_{j}^{\perp}, \mathbf{z}^{T} Q_{i} \mathbf{z}_{j}\right)
\end{aligned}
$$

with $Q_{i}$ given in (15), and $\mathbf{z}_{j}^{\perp}$ is defined as

$$
\mathbf{z}_{j}^{\perp} \triangleq \check{R}\left(\frac{\pi}{2}, \frac{\pi}{2}, \frac{\pi}{2}\right) \mathbf{z}_{j}
$$

with

$$
\mathbf{z}_{0}=\left[\begin{array}{c}
\mathbf{z}_{f}^{0} \\
\hline 0 \\
\hline 0 \\
\hline \mathbf{o}_{1}^{x y} \\
\hline 0
\end{array}\right], \mathbf{z}_{1}=\left[\begin{array}{c}
0 \\
\hline \mathbf{z}_{f}^{1} \\
\hline 0 \\
\hline 0 \\
\hline \mathbf{o}_{2}^{x y}
\end{array}\right], \mathbf{z}_{2}=\left[\begin{array}{c}
0 \\
\hline 0 \\
\hline \mathbf{z}_{f}^{2} \\
\hline 0 \\
\hline 0
\end{array}\right]
$$

Proof. Firstly, it can be readily proved by using Theorem 1 of (Wang et al. 2015) that minimizing the objective function $F(\mathbf{x})$ in (6) is equivalent to minimizing

$$
\begin{gathered}
f_{0}\left(\phi_{r_{1}}, \phi_{r_{2}}\right)=c_{0}-2 \sum_{0 \leq i<2} \sum_{i<j \leq 2} b_{i j} \cos \left(\phi_{r_{j}}-\phi_{r_{i}}-\phi_{i j 0}\right) \\
+\sum_{i=1}^{2} \frac{\gamma_{i}}{2}\left\|R\left(o_{i}^{\phi}\right)-R^{T}\left(\phi_{r_{i-1}}\right) R\left(\phi_{r_{i}}\right)\right\|_{F}^{2}
\end{gathered}
$$

where $c_{0} \triangleq \mathbf{z}^{T} C \mathbf{z}-\sum_{i=0}^{2} b_{i i}$. Note that

$$
\begin{aligned}
& R\left(o_{i}^{\phi}\right)-R^{T}\left(\phi_{r_{i-1}}\right) R\left(\phi_{i}\right)= \\
& {\left[\begin{array}{cc}
\cos \left(o_{i}^{\phi}\right)-\cos \left(\phi_{r_{i}}-\phi_{r_{i-1}}\right) & -\sin \left(o_{i}^{\phi}\right)+\sin \left(\phi_{r_{i}}-\phi_{r_{i-1}}\right) \\
\sin \left(o_{i}^{\phi}\right)-\sin \left(\phi_{r_{i}}-\phi_{r_{i-1}}\right) & \cos \left(o_{i}^{\phi}\right)-\cos \left(\phi_{r_{i}}-\phi_{r_{i-1}}\right)
\end{array}\right]}
\end{aligned}
$$


Thus, we have

$\left\|R\left(o_{i}^{\phi}\right)-R^{T}\left(\phi_{r_{i-1}}\right) R\left(\phi_{i}\right)\right\|_{F}^{2}=4-4 \cos \left(\phi_{r_{i}}-\phi_{r_{i-1}}-o_{i}^{\phi}\right)$.

Substitute the above result into (21), the proof is evident.

\section{2 m-step SLAM}

For the $m$-step case (5), we have the following theorem:

Theorem 2: The $m$-step SLAM problem (minimizing the objective function $F(\mathbf{x})$ in (5)) is equivalent to minimizing the following objective function with only $m$ variables $\phi_{r_{1}}, \cdots, \phi_{r_{m}}$

$$
\begin{aligned}
& f_{0}\left(\phi_{r_{1}}, \cdots, \phi_{r_{m}}\right) \\
= & c_{1}-2 \sum_{0 \leq i<j \leq m} b_{i j} \cos \left(\phi_{r_{j}}-\phi_{r_{i}}-\phi_{i j 0}\right) \\
& -2 \sum_{i=1}^{m} \gamma_{i} \cos \left(\phi_{r_{i}}-\phi_{r_{i-1}}-o_{i}^{\phi}\right)
\end{aligned}
$$

where

$$
c_{1}=\mathbf{z}^{T} C \mathbf{z}-\sum_{i=0}^{m} b_{i i}+2 \sum_{i=1}^{m} \gamma_{i}
$$

and for $i, j=0, \ldots, m$,

$$
\begin{aligned}
b_{i j} & \left.=\sqrt{\left(\mathbf{z}^{T} Q_{i} \mathbf{z}_{j}\right)^{2}+\left(\mathbf{z}^{T} Q_{i} \mathbf{z}_{j}^{\perp}\right.}\right)^{2} \\
\phi_{i j 0} & =\operatorname{atan} 2\left(\mathbf{z}^{T} Q_{i} \mathbf{z}_{j}^{\perp}, \mathbf{z}^{T} Q_{i} \mathbf{z}_{j}\right)
\end{aligned}
$$

with $Q_{i}=C A_{i}^{T}\left(A C A^{T}\right)^{-1} A C$, where $A, A_{i}, C, \mathbf{z}, \mathbf{z}_{j}$ are similar to those defined in Section 4.1.

Proof. The proof of Theorem 2 is similar to that of Theorem 1 and is omitted here.

Corollary 1: The $m$-step SLAM problem (22) is equivalent to minimizing

$$
\begin{aligned}
& f_{0}\left(\phi_{r_{1}}, \cdots, \phi_{r_{m}}\right) \\
= & c_{1}-2 \sum_{0 \leq i<j \leq m} \beta_{i j} \cos \left(\phi_{r_{j}}-\phi_{r_{i}}-\varphi_{i j 0}\right)
\end{aligned}
$$

where $\beta_{i j}=b_{i j}, \varphi_{i j 0}=\phi_{i j 0}$ except for $i=0, \ldots, m-$ $1, j=i+1$ such that

$$
\begin{aligned}
\beta_{i j} & =\sqrt{b_{i j}^{2}+\gamma_{j}^{2}+2 b_{i j} \gamma_{j} \cos \left(\phi_{i j 0}-o_{j}^{\phi}\right)} \\
\varphi_{i j 0} & =\operatorname{atan} 2\left(b_{i j} \sin \phi_{i j 0}+\gamma_{j} \sin o_{j}^{\phi}, b_{i j} \cos \phi_{i j 0}+\gamma_{j} \cos o_{j}^{\phi}\right)
\end{aligned}
$$

and $c_{1}$ is defined in (23).
Proof. Denote $\tilde{\phi}_{i j}^{f}=\phi_{r_{j}}-\phi_{r_{i}}-\phi_{i j 0}$, we have that

$$
\begin{aligned}
& -2 \sum_{0 \leq i<j \leq m} b_{i j} \cos \tilde{\phi}_{i j}^{f}-2 \sum_{i=1}^{m} \gamma_{i} \cos \left(\phi_{r_{i}}-\phi_{r_{i-1}}-o_{i}^{\phi}\right) \\
& =-2 \sum_{\substack{0 \leq i \leq m-1 \\
j=i+1}} b_{i j} \cos \tilde{\phi}_{i j}^{f}-2 \sum_{\substack{0 \leq i \leq m-1 \\
i+1<j \leq m}} b_{i j} \cos \tilde{\phi}_{i j}^{f} \\
& -2 \sum_{i=1}^{m} \gamma_{i} \cos \left(\phi_{r_{i}}-\phi_{r_{i-1}}-o_{i}^{\phi}\right) \\
& =-2 \sum_{\substack{0 \leq i \leq m-1 \\
j=i+1}}\left(b_{i j} \cos \tilde{\phi}_{i j}^{f}+\gamma_{j} \cos \left(\phi_{r_{j}}-\phi_{r_{i}}-o_{j}^{\phi}\right)\right) \\
& -2 \sum_{\substack{0 \leq i \leq m-1 \\
i+1<j \leq m}} b_{i j} \cos \tilde{\phi}_{i j}^{f} \\
& =-2 \sum_{0 \leq i<j \leq m-1} \beta_{i j} \cos \tilde{\phi}_{i j}^{f} \text {. }
\end{aligned}
$$

Substitute the above equation into (22), the proof is evident.

Remark 1: From Corollary 1, it can be seen that the format of orientation part is similar to the $x-y$ part if we use Frobenius norm in the objective function (5), this is different from the case when objective function (3) is used (Wang et al. 2015).

\section{Alternative form of primal unconstrained SLAM op- timization problem}

In this section, we convert the unconstrained optimization problem of the $m$-step SLAM problem as described in Corollary 1 into a constrained optimization, which is essential for the analysis of globally optimal solution.

Corollary 2: The $m$-step SLAM problem (26) is equivalent to the following optimization problem

$$
\begin{aligned}
& \min c_{2}+\sum_{0 \leq i<j \leq m} \beta_{i j}\left\|\mathbf{p}_{j}-R_{i j} \mathbf{p}_{i}\right\|_{2}^{2} \\
& \text { s.t., }\left\|\mathbf{p}_{i}\right\|_{2}^{2}=1, \quad i=0, \ldots, m
\end{aligned}
$$

where $\mathbf{p}_{i}=\left[\cos \phi_{r_{i}} \sin \phi_{r_{i}}\right]^{T}$ for $i=0, \ldots, m$ with $\mathbf{p}_{0}=$ $\left[\begin{array}{ll}1 & 0\end{array}\right]^{T}$, and

$$
R_{i j}=\left[\begin{array}{cc}
\cos \varphi_{i j 0} & -\sin \varphi_{i j 0} \\
\sin \varphi_{i j 0} & \cos \varphi_{i j 0}
\end{array}\right], c_{2}=c_{1}-2 \sum_{0 \leq i<j \leq m} \beta_{i j}
$$


Proof. Note that

$$
\begin{aligned}
& \left\|\mathbf{p}_{j}-R_{i j} \mathbf{p}_{i}\right\|_{2}^{2} \\
= & \left\|\left[\begin{array}{l}
\cos \phi_{r_{j}} \\
\sin \phi_{r_{j}}
\end{array}\right]-\left[\begin{array}{cc}
\cos \varphi_{i j 0} & -\sin \varphi_{i j 0} \\
\sin \varphi_{i j 0} & \cos \varphi_{i j 0}
\end{array}\right]\left[\begin{array}{c}
\cos \phi_{r_{i}} \\
\sin \phi_{r_{i}}
\end{array}\right]\right\|_{2}^{2} \\
= & \left\|\left[\begin{array}{c}
\cos \phi_{r_{j}}-\cos \left(\varphi_{i j 0}+\phi_{r_{i}}\right) \\
\sin \phi_{r_{j}}-\sin \left(\varphi_{i j 0}+\phi_{r_{i}}\right)
\end{array}\right]\right\|_{2}^{2} \\
= & \left(\cos \phi_{r_{j}}-\cos \left(\varphi_{i j 0}+\phi_{r_{i}}\right)\right)^{2}+\left(\sin \phi_{r_{j}}-\sin \left(\varphi_{i j 0}+\phi_{r_{i}}\right)\right)^{2} \\
= & 2-2 \cos \left(\phi_{r_{j}}-\phi_{r_{i}}-\varphi_{i j 0}\right) .
\end{aligned}
$$

Thus,

$$
\beta_{i j}\left\|\mathbf{p}_{j}-R_{i j} \mathbf{p}_{i}\right\|_{2}^{2}=2 \beta_{i j}-2 \beta_{i j} \cos \left(\phi_{r_{j}}-\phi_{r_{i}}-\varphi_{i j 0}\right) .
$$

Substitute above equation into (26), the proof is evident.

Optimization problem (27) is equivalent to

$$
\begin{array}{ll}
\min & \overline{\mathbf{p}}^{T} W_{0} \overline{\mathbf{p}}+c_{2} \\
\text { s.t., } & \mathbf{p}_{i}^{T} \mathbf{p}_{i}=1, i=1, \ldots, m
\end{array}
$$

where $\overline{\mathbf{p}}=\left[\begin{array}{lllll}\mathbf{p}_{m}^{T} & \mathbf{p}_{m-1}^{T} & \cdots & \mathbf{p}_{1}^{T} & \mathbf{p}_{0}^{T}\end{array}\right]^{T}$, and $W_{0}$ is a $2(m+$ 1) $\times 2(m+1)$ matrix which can be denoted as

$$
W_{0}=\left[\begin{array}{ccc}
\alpha_{m m} I_{2} & -\beta_{(m-1) m} R_{(m-1) m} & \cdots \\
-\beta_{(m-1) m} R_{(m-1) m}^{T} & \alpha_{(m-1)(m-1)} I_{2} & \cdots \\
\vdots & \vdots & \cdots \\
-\beta_{1 m} R_{1 m}^{T} & -\beta_{1(m-1)} R_{1(m-1)}^{T} & \cdots \\
-\beta_{0 m} R_{0 m}^{T} & -\beta_{0(m-1)} R_{0(m-1)}^{T} & \cdots \\
-\beta_{1 m} R_{1 m} & -\beta_{0 m} R_{0 m} \\
-\beta_{1(m-1)} R_{1(m-1)} & -\beta_{0(m-1)} R_{0(m-1)} \\
\vdots & \vdots \\
\alpha_{11} I_{2} & -\beta_{01} R_{01} \\
-\beta_{01} R_{01}^{T} & \alpha_{00} I_{2}
\end{array}\right]
$$

where for $i=0, \ldots, m$,

$$
\alpha_{i i}=\sum_{k \neq i} \beta_{k i}
$$

with $\beta_{k i}=\beta_{i k}$.

Notice that since $\mathbf{p}_{0}=\left[\begin{array}{ll}1 & 0\end{array}\right]^{T}$, we can remove the column and row of $W_{0}$ which corresponds to 0 , such that (28) becomes

$$
\begin{aligned}
& \min \mathbf{p}^{T} W \mathbf{p}+c_{2} \\
& \text { s.t., } \mathbf{p}_{i}^{T} \mathbf{p}_{i}=1, i=1, \ldots, m
\end{aligned}
$$

where $\mathbf{p}=\left[\begin{array}{lllll}\mathbf{p}_{m}^{T} \mathbf{p}_{m-1}^{T} & \cdots & \mathbf{p}_{1}^{T} & 1\end{array}\right]^{T}$, and

$$
W=\left[\begin{array}{ccc}
\alpha_{m m} I_{2} & -\beta_{(m-1) m} R_{(m-1) m} & \cdots \\
-\beta_{(m-1) m} R_{(m-1) m}^{T} & \alpha_{(m-1)(m-1)} I_{2} & \cdots \\
\vdots & \vdots & \cdots \\
-\beta_{1 m} R_{1 m}^{T} & -\beta_{1(m-1)} R_{1(m-1)}^{T} & \cdots \\
-\beta_{0 m} \mathbf{v}_{0 m}^{T} & -\beta_{0(m-1)} \mathbf{v}_{0(m-1)}^{T} & \cdots \\
& & \\
-\beta_{1 m} R_{1 m} & -\beta_{0 m} \mathbf{v}_{0 m} \\
-\beta_{1(m-1)} R_{1(m-1)} & -\beta_{0(m-1)} \mathbf{v}_{0(m-1)} \\
\vdots & \vdots \\
\alpha_{11} I_{2} & -\beta_{01} \mathbf{v}_{01} \\
-\beta_{01} \mathbf{v}_{01}^{T} & \alpha_{00}
\end{array}\right]
$$

with $\mathbf{v}_{0 k}=\left[\begin{array}{ll}\cos \varphi_{0 k 0} & \sin \varphi_{0 k 0}\end{array}\right]^{T}$ for $k=1, \cdots, m$.

Particularly, for one-step case, i.e., $m=1$, we have $\mathbf{p}=$ $\left[\mathbf{p}_{1}^{T} 1\right]^{T}$, and

$$
W=\left[\begin{array}{cc}
\beta_{01} I_{2} & -\beta_{01} \mathbf{v}_{01} \\
-\beta_{01} \mathbf{v}_{01}^{T} & \beta_{01}
\end{array}\right]=\beta_{01}\left[\begin{array}{cc}
I_{2} & -\mathbf{v}_{01} \\
-\mathbf{v}_{01}^{T} & 1
\end{array}\right]
$$

and for two-step case, i.e., $m=2$, we have $\mathbf{p}=\left[\begin{array}{l}\mathbf{p}_{2}^{T} \mathbf{p}_{1}^{T} 1 \\ 1\end{array}\right]^{T}$,

$$
W=\left[\begin{array}{ccc}
\left(\beta_{12}+\beta_{02}\right) I_{2} & -\beta_{12} R_{12} & -\beta_{02} \mathbf{v}_{02} \\
-\beta_{12} R_{12}^{T} & \left(\beta_{12}+\beta_{01}\right) I_{2} & -\beta_{01} \mathbf{v}_{01} \\
-\beta_{02} \mathbf{v}_{02}^{T} & -\beta_{01} \mathbf{v}_{01}^{T} & \beta_{01}+\beta_{02}
\end{array}\right]
$$

\section{Globally optimal solution to one-step SLAM}

In this section, the globally optimal solution to one-step SLAM is studied, through investigating the strong duality condition, and an example is introduced to verify the result.

\subsection{Globally optimal solution to primal problem}

In (5), when $m=1$, the problem becomes one-step SLAM, and from Theorem 1, it can be seen that the one-step SLAM problem is equivalent to minimizing the following objective function:

$$
\begin{aligned}
f_{0}\left(\phi_{r_{1}}\right) & =c_{1}-2 b_{01} \cos \left(\phi_{r_{1}}-\phi_{010}\right)-2 \gamma_{1} \cos \left(\phi_{r_{1}}-o_{1}^{\phi}\right) \\
& =c_{1}-2 \beta_{01} \cos \left(\phi_{r_{1}}-\varphi_{010}\right)
\end{aligned}
$$


where $c_{1}=\mathbf{z}^{T} C \mathbf{z}-\sum_{i=0}^{1} b_{i i}+2 \gamma_{1}$, and

$$
\begin{aligned}
& \beta_{01}= \sqrt{b_{01}^{2}+\gamma_{1}^{2}+2 b_{01} \gamma_{1} \cos \left(\phi_{010}-o_{1}^{\phi}\right)} \\
& \varphi_{010}=\operatorname{atan} 2\left(b_{01} \sin \phi_{010}+\gamma_{1} \sin o_{1}^{\phi}\right. \\
&\left.b_{01} \cos \phi_{010}+\gamma_{1} \cos o_{1}^{\phi}\right) .
\end{aligned}
$$

Consider interval $[-\pi, \pi)$, there is only one minimum, i.e., $\phi_{r_{1}}-\varphi_{010}=0$ which means the globally optimal solution to one-step SLAM is

$$
\phi_{r_{1}}=\varphi_{010}
$$

Thus, the global minimum of $f_{0}\left(\phi_{r_{1}}\right)$ is

$$
f_{0}\left(\phi_{r_{1}}^{*}\right)=c_{1}-2 \beta_{01}
$$

\subsection{Strong duality for one-step case}

For one-step SLAM case, the primal problem can be rewritten as

$$
\begin{aligned}
& \min \mathbf{p}^{T} W \mathbf{p}+c_{2} \\
& \text { s.t., } \mathbf{p}_{1}^{T} \mathbf{p}_{1}=1
\end{aligned}
$$

where $\mathbf{p}=\left[\begin{array}{ll}\mathbf{p}_{1}^{T} & 1\end{array}\right]^{T}$, and

$$
c_{2}=\mathbf{z}^{T} C \mathbf{z}-\sum_{i=0}^{1} b_{i i}+2 \gamma_{1}-2 \beta_{01}
$$

From the analysis in Section 6.1, we have known that the global minimum of the primal problem (28) when $m=1$ is

$$
\begin{aligned}
f^{*} & =c_{1}-2 \beta_{01} \\
& =\mathbf{z}^{T} C \mathbf{z}-\sum_{i=0}^{1} b_{i i}+2 \gamma_{1}-2 \beta_{01} \\
& =c_{2}
\end{aligned}
$$

where $c_{1}=\mathbf{z}^{T} C \mathbf{z}-\sum_{i=0}^{1} b_{i i}+2 \gamma_{1}$, and the optimal solution is $\phi_{r_{1}}=\varphi_{010}$. Next, we will calculate the global minimum of the Lagrangian dual problem.

The Lagrangian of primal problem is

$$
L\left(p_{1}, \bar{\lambda}_{1}\right)=\mathbf{p}^{T} W \mathbf{p}+c_{2}+\bar{\lambda}_{1}\left(1-\mathbf{p}_{1}^{T} \mathbf{p}_{1}\right)
$$

where $\bar{\lambda}_{1}$ is Lagrange multiplier.

Let $\bar{\lambda}_{1}=\beta_{01} \lambda_{1}$, the dual function $\mathrm{d}: \mathbb{R}^{n} \longrightarrow \mathbb{R}$ is the infimum of the Lagrangian with respect to $\mathbf{p}_{1}$ :

$$
d\left(\lambda_{1}\right)=\inf _{\mathbf{p}_{1}} L\left(\mathbf{p}_{1}, \lambda_{1}\right)=\inf _{\mathbf{p}_{1}} \mathbf{p}^{T} \tilde{W} \mathbf{p}+c_{2}+\beta_{01} \lambda_{1}
$$

where

$$
\tilde{W}=\beta_{01}\left[\begin{array}{cc}
\left(1-\lambda_{1}\right) I & -\mathbf{v}_{01} \\
-\mathbf{v}_{01}^{T} & 1
\end{array}\right]
$$

The Lagrangian dual problem is to look for a maximum of the dual function over $\lambda_{1}$ :

$$
d^{*}=\max _{\lambda_{1}} d\left(\lambda_{1}\right)=\max _{\lambda_{1}} \inf _{\mathbf{p}_{1}} \mathbf{p}^{T} \tilde{W} \mathbf{p}+c_{2}+\beta_{01} \lambda_{1} .
$$

Set $L\left(\mathbf{p}_{1}, \lambda_{1}\right)$ 's partial derivative with respect to $\mathbf{p}_{1}$ to zero:

$$
\frac{\partial L\left(\mathbf{p}_{1}, \lambda_{1}\right)}{\partial \mathbf{p}_{1}}=0
$$

which generates

$$
\frac{\partial L\left(\mathbf{p}_{1}, \lambda_{1}\right)}{\partial \mathbf{p}_{1}}=2\left(1-\lambda_{1}\right) \mathbf{p}_{1}-2 \mathbf{v}_{01}=0
$$

From (40), we have $\mathbf{p}_{1}=\frac{1}{1-\lambda_{1}} \mathbf{v}_{01}$, substitute it into (38), we have

$$
d^{*}=\max _{\lambda_{1}} d\left(\lambda_{1}\right)=\max _{\lambda_{1}} \beta_{01}\left(-\frac{1}{1-\lambda_{1}}+1\right)+c_{2}+\beta_{01} \lambda_{1}
$$

The maximum of $d\left(\lambda_{1}\right)$ can be obtained by solving

$$
\frac{\partial d\left(\lambda_{1}\right)}{\partial \lambda_{1}}=\beta_{01}-\frac{\beta_{01}}{\left(1-\lambda_{1}\right)^{2}}=0
$$

from which we have $\lambda_{1}^{*}=0$, thus, the optimal minimum of the Lagrangian dual

$$
d^{*}=c_{2}
$$

with the optimal solution $\mathbf{p}_{1}=\mathbf{v}_{01}$, thus, $\phi_{r_{1}}=\varphi_{010}$, which equals the optimal solution to the primal problem. Compare $d^{*}$ in (43) with $f^{*}$ in (36) of the primal problem, we have $d^{*}=f^{*}$, the so called strong duality holds.

Remark 2: It can be concluded that no matter what level noise is, for one-step SLAM here, the optimal solution to the Lagrangian dual problem (37) is equal to that of primal optimal problem (35), and this solution is unique. Furthermore, it can be proved that the eigenvalues of $\tilde{W}\left(\lambda^{*}\right)$ for the Lagrangian dual problem (37) are

$$
1-\lambda_{1}^{*}, \frac{2-\lambda_{1}^{*} \pm \sqrt{\lambda_{1}^{*^{2}}+4}}{2}
$$

From (42), we have that $\lambda_{1}^{*}=0$, thus, there is only one eigenvalue which satisfies the Single Zero Eigenvalue Property (SZEP) condition proposed in (Carlone et al. 2016). 
Remark 3: Through using objective function (5) for onestep case, there exists only one minimum which is global optimal, however, applying objective function (3), according to the result obtained in (Wang et al. 2013), when some conditions as proposed in Theorem 2 of (Wang et al. 2013) are satisfied, there are two local minima. The following Example 1 shows the advantage of using objective function (5) instead of (3) for one-step case.

Example 1: Consider one-step one feature case, given ground truth of robot pose $r_{1}$ and feature $f_{1}$ as $\mathbf{x}_{r_{1}}=\left[\begin{array}{lll}1 & 5 & 0.2\end{array}\right]^{T}$ and $\mathbf{x}_{f_{1}}=\left[\begin{array}{ll}-5 & 5\end{array}\right]^{T}$, respectively. Let the observation and odometry be affected by noises with mean 0 and standard deviation 0.7 , which are $\mathbf{z}_{f}^{0}=\left[\begin{array}{ll}-5.3488 & 5.2851\end{array}\right]^{T}, \mathbf{z}_{f}^{1}=\left[\begin{array}{ll}-5.8028 & 0.9995\end{array}\right]^{T}$, $\mathbf{o}_{1}^{x y}=\left[\begin{array}{ll}1.0605 & 5.3026\end{array}\right]^{T}, o_{1}^{\phi}=3.1610$. If we use objective function (3), the one-step SLAM problem can be converted into solving (10) of (Wang et al. 2013), and from Theorem 2 of (Wang et al. 2013), it can be seen that conditions (16)(20) of (Wang et al. 2013) are satisfied, there are two minima, i.e., $\mathbf{x}=\left[\begin{array}{lllll}-5.1259 & 4.8542 & 0.8376 & 5.7335 & 0.395\end{array}\right)^{T}$ and $\mathbf{x}=\left[\begin{array}{lllll}-5.1178 & 5.7617 & 0.8295 & 4.8260 & -0.0716\end{array}\right]^{T}$, where $\mathbf{x}=\left[\begin{array}{lll}\mathbf{x}_{f_{1}}^{T} & \mathbf{x}_{r_{1}}^{T} & \phi_{r_{1}}\end{array}\right]^{T}$. However, if we use objective function (5), from above analysis, we have that there is only one minimum which is the global optimal solution to the one step SLAM problem, i.e., $\mathbf{x}=\left[\begin{array}{lllll}-5.1749 & 5.2597 & 0.8865 & 5.3281 & 0.1865\end{array}\right]^{T}$.

\section{Globally optimal solution to primal two-step SLAM}

In this section, conditions to obtain the globally optimal solution of the two-step SLAM case are drawn through investigating the corresponding Lagrangian dual problem, an example is provided to verify the main theorem of this section.

Similar to one-step case, the Lagrangian dual function $d$ is the infimum of Lagrangian with respect to $\mathbf{p}_{2}, \mathbf{p}_{1}$ :

$d\left(\lambda_{1}, \lambda_{2}\right)=\inf _{\mathbf{p}} L(\mathbf{p}, \lambda)=\inf _{\mathbf{p}_{2}, \mathbf{p}_{1}} \mathbf{p}^{T} W(\lambda) \mathbf{p}+c_{2}+\sum_{i=1}^{2} \lambda_{i}$

where $\mathbf{p}=\left[\begin{array}{lll}\mathbf{p}_{2}^{T} & \mathbf{p}_{1}^{T} & 1\end{array}\right]^{T}, c_{2}=\mathbf{z}^{T} C \mathbf{z}-\sum_{i=0}^{1} b_{i i}+$ $2 \sum_{i=1}^{2} \gamma_{i}-2 \sum_{0 \leq i<j \leq 2} \beta_{i j}$

$$
W(\lambda)=\left[\begin{array}{ccc}
W_{11}(\lambda) & -\beta_{12} R_{12} & -\beta_{02} \mathbf{v}_{02} \\
-\beta_{12} R_{12}^{T} & W_{22}(\lambda) & -\beta_{01} \mathbf{v}_{01} \\
-\beta_{02} \mathbf{v}_{02}^{T} & -\beta_{01} \mathbf{v}_{01}^{T} & w_{33}
\end{array}\right]
$$

where $W_{11}(\lambda)=\left(\beta_{12}+\beta_{02}-\lambda_{2}\right) I_{2}, W_{22}(\lambda)=\left(\beta_{12}+\right.$ $\left.\beta_{01}-\lambda_{1}\right) I_{2}, w_{33}=\beta_{01}+\beta_{02}$. The following lemma is borrowed from (Gallier 2010).

Lemma 1: If $W$ is a symmetric matrix, then the function

$$
f(\mathbf{p})=\mathbf{p}^{T} W \mathbf{p}+2 \mathbf{p}^{T} \mathbf{u}
$$

has a minimum value iff $W \geq 0$ and $\left(I-W W^{\dagger}\right) \mathbf{u}=0$, in which case this minimum value is

$$
f^{*}=-\mathbf{u}^{T} W^{\dagger} \mathbf{u}
$$

The lagrangian dual problem is to look for a maximum of the dual function over $\lambda$ :

$$
\begin{aligned}
d^{*} & =\max _{\lambda} d(\lambda)=\max _{\lambda} \inf _{\mathbf{p}} \mathbf{p}^{T} W(\lambda) \mathbf{p}+c_{2}+\sum_{i=1}^{2} \lambda_{i} \\
& =\max _{\lambda} \inf _{\mathbf{p}} \mathbf{p}^{T} \tilde{W}(\lambda) \mathbf{p}+2 \mathbf{p}^{T} \mathbf{u}+v+c_{2}+\sum_{i=1}^{2} \lambda_{i}
\end{aligned}
$$

where

$$
\begin{aligned}
& W(\lambda)=\left[\begin{array}{cc}
\tilde{W}(\lambda) & \mathbf{u} \\
\mathbf{u}^{T} & v
\end{array}\right], \mathbf{u}=\left[\begin{array}{c}
-\beta_{02} \mathbf{v}_{02} \\
-\beta_{01} \mathbf{v}_{01}
\end{array}\right], v=\beta_{01}+\beta_{02} \\
& \tilde{W}(\lambda)=\left[\begin{array}{cc}
\left(\beta_{12}+\beta_{02}-\lambda_{2}\right) I_{2} & -\beta_{12} R_{12} \\
-\beta_{12} R_{12}^{T} & \left(\beta_{12}+\beta_{01}-\lambda_{1}\right) I_{2}
\end{array}\right] \text {. }
\end{aligned}
$$

Since we consider the case when there exists minimum, apply Lemma 1, we have

$$
d^{*}=\max _{\lambda_{2}, \lambda_{1}}-\mathbf{u}^{T} \tilde{W}(\lambda)^{\dagger} \mathbf{u}+v+c_{2}+\sum_{i=1}^{2} \lambda_{i} .
$$

Using Schur complement lemma, the problem can be converted into

$$
\begin{gathered}
d^{*}=\max _{\lambda_{2}, \lambda_{1}} \lambda_{0}+c_{2}+\sum_{i=1}^{2} \lambda_{i} \\
\text { s.t., } \bar{W}(\lambda) \geq 0
\end{gathered}
$$

where $\bar{W}(\lambda)=\left[\begin{array}{cc}\tilde{W}(\lambda) & -\mathbf{u} \\ -\mathbf{u}^{T} & v-\lambda_{0}\end{array}\right]$, which is equivalent to

$$
\begin{gathered}
d^{*}=\max _{\lambda} \sum_{i=0}^{2} \lambda_{i}+c_{2} \\
\text { s.t., } \bar{W}(\lambda) \geq 0
\end{gathered}
$$

where $\lambda=\left[\begin{array}{lll}\lambda_{2} & \lambda_{1} & \lambda_{0}\end{array}\right]^{T}$, and

$$
\bar{W}(\lambda)=\left[\begin{array}{ccc}
\bar{W}_{11}(\lambda) & -\beta_{12} R_{12} & -\beta_{02} \mathbf{v}_{02} \\
-\beta_{12} R_{12}^{T} & \bar{W}_{22}(\lambda) & -\beta_{01} \mathbf{v}_{01} \\
-\beta_{02} \mathbf{v}_{02}^{T} & -\beta_{01} \mathbf{v}_{01}^{T} & \bar{W}_{33}(\lambda)
\end{array}\right]
$$


where $\bar{W}_{11}(\lambda)=\left(\beta_{12}+\beta_{02}-\lambda_{2}\right) I_{2}, \bar{W}_{22}(\lambda)=\left(\beta_{12}+\right.$ $\left.\beta_{01}-\lambda_{1}\right) I_{2}, \bar{W}_{33}(\lambda)=\beta_{01}+\beta_{02}-\lambda_{0}$.

Optimization problem (48) is a convex optimization problem which can be solved efficiently using the Matlab LMI toolbox. The following two lemmas can be proved by following similar lines as proposed in (Carlone et al. 2016) which is omitted in this paper.

Lemma 2: The optimal value $d^{*}$ in (48) is attained at a finite $\lambda$. Moreover, the penalized matrix $\bar{W}\left(\lambda^{*}\right)$ has one zero eigenvalue.

Lemma 3: If the zero eigenvalue of the penalized matrix $\bar{W}\left(\lambda^{*}\right)$ is simple, then the duality gap is zero, i.e., $d^{*}=f^{*}$.

From Lemma 2, we have that $\bar{W}\left(\lambda^{*}\right)$ at least has one zero eigenvalue, in the following, we prove that for two-step case, i.e., $m=2$, there is only one zero eigenvalue.

The following two lemmas are common which will be used in later development.

Lemma 4: If matrices $A, B$ satisfy

$$
\left[\begin{array}{cc}
A & B \\
B^{T} & 0
\end{array}\right] \geq 0
$$

then $A \geq 0$ and $B=0$.

Lemma 5: Multiplication by nonsingular matrices cannot change rank, e.g., given elementary matrix $P$, the rank of $\mathcal{W}(\lambda)=P \bar{W}(\lambda) P^{T}$ is the same as that of $\bar{W}(\lambda)$. In addition, if $\bar{W}(\lambda) \geq 0$, we have $\mathcal{W}(\lambda) \geq 0$ also holds.

The main result for two-step SLAM is given in the following theorem:

Theorem 3: Given solution $\lambda^{*}$ to Lagrangian dual problem (48), $\bar{W}\left(\lambda^{*}\right)$ always has only one zero eigenvalue except the following conditions are satisfied simultaneously

$$
\begin{aligned}
& \varphi_{020}-\varphi_{010}-\varphi_{120}=(2 k+1) \pi, k \in\{-2,-1,0,1\} \\
& \lambda_{2}=\beta_{12}+\beta_{02}-\frac{\beta_{02} \beta_{12}}{\beta_{01}} \\
& \lambda_{1}=\beta_{12}+\beta_{01}-\frac{\beta_{01} \beta_{12}}{\beta_{02}}
\end{aligned}
$$

Further, when $\bar{W}\left(\lambda^{*}\right)$ has only one zero eigenvalue, the duality gap is zero, i.e., $d^{*}=f^{*}$.

Proof. From Lemma 4, it can be concluded that $\beta_{01}+\beta_{02}-$ $\lambda_{0}^{*}>0$ since $\mathbf{v}_{02} \neq 0, \mathbf{v}_{01} \neq 0$. Similarly, we have $\beta_{12}+$ $\beta_{02}-\lambda_{2}^{*}>0$ and $\beta_{12}+\beta_{01}-\lambda_{1}^{*}>0$.
Firstly, through elementary transformation, i.e., multiply both the left and right sides of $\bar{W}(\lambda)$ by

$$
P_{1}=\left[\begin{array}{ccc}
I_{2} & 0 & 0 \\
\frac{\beta_{12} R_{12}^{T}}{\beta_{12}+\beta_{02}-\lambda_{2}^{*}} & I_{2} & 0 \\
\frac{\beta_{02} \mathbf{v}_{02}^{T}}{\beta_{12}+\beta_{02}-\lambda_{2}^{*}} & 0 & I_{2}
\end{array}\right]
$$

and $P_{1}^{T}$, respectively, we can convert $\bar{W}\left(\lambda^{*}\right)$ into

$$
\mathcal{W}\left(\lambda^{*}\right)=\left[\begin{array}{ccc}
\mathcal{W}_{11}\left(\lambda^{*}\right) & 0 & 0 \\
0 & \mathcal{W}_{22}\left(\lambda^{*}\right) & -\mathbf{b} \\
0 & -\mathbf{b}^{T} & \mathcal{W}_{33}\left(\lambda^{*}\right)
\end{array}\right]
$$

where $\mathcal{W}_{11}\left(\lambda^{*}\right)=\left(\beta_{12}+\beta_{02}-\lambda_{2}^{*}\right) I_{2}, \mathcal{W}_{22}\left(\lambda^{*}\right)=\left(\beta_{12}+\right.$ $\left.\beta_{01}-\lambda_{1}^{*}-\frac{\beta_{12}^{2}}{\beta_{12}+\beta_{02}-\lambda_{2}^{*}}\right) I_{2}, \mathcal{W}_{33}\left(\lambda^{*}\right)=\beta_{01}+\beta_{02}-\lambda_{0}-$ $\frac{\beta_{02}^{2}}{\beta_{12}+\beta_{02}-\lambda_{2}^{*}}, \mathbf{b}^{T}=\left(\beta_{01} \mathbf{v}_{01}^{T}+\frac{\beta_{02} \beta_{12} \mathbf{v}_{02}^{T} R_{12}}{\beta_{12}+\beta_{02}-\lambda_{2}^{*}}\right)$, apply Lemma 5 , we have that the number of zero eigenvalue of $\mathcal{W}\left(\lambda^{*}\right)$ is equal to that of $\bar{W}\left(\lambda^{*}\right)$, and

$$
\mathcal{W}\left(\lambda^{*}\right) \geq 0
$$

and there exists at least one zero eigenvalue for $\mathcal{W}\left(\lambda^{*}\right)$, which means

$$
\begin{gathered}
{\left[\begin{array}{c}
\left(\beta_{12}+\beta_{01}-\lambda_{1}^{*}-\frac{\beta_{12}^{2}}{\beta_{12}+\beta_{02}-\lambda_{2}^{*}}\right) I_{2} \\
-\mathbf{b}^{T} \\
-\mathbf{b} \\
\beta_{01}+\beta_{02}-\lambda_{0}^{*}-\frac{\beta_{02}^{2}}{\beta_{12}+\beta_{02}-\lambda_{2}^{*}}
\end{array}\right] \geq 0}
\end{gathered}
$$

If $\mathbf{b} \neq 0$, from Lemma 4 , it can be concluded that

$$
\beta_{12}+\beta_{01}-\lambda_{1}^{*}-\frac{\beta_{12}^{2}}{\beta_{12}+\beta_{02}-\lambda_{2}^{*}}>0 .
$$

Multiply both the left and right sides of $\mathcal{W}\left(\lambda^{*}\right)$ in (51) by

$$
P_{2}=\left[\begin{array}{ccr}
I_{2} & 0 & 0 \\
0 & I_{2} & 0 \\
0 & \mathbf{b}^{T} & I_{2} \\
\beta_{12}+\beta_{01}-\lambda_{1}^{*}-\frac{\beta_{12}^{2}}{\beta_{12}+\beta_{02}-\lambda_{2}^{*}} &
\end{array}\right]
$$

and $P_{2}^{T}$, respectively, we can convert $\mathcal{W}\left(\lambda^{*}\right)$ into

$$
\mathcal{W}\left(\lambda^{*}\right)=\left[\begin{array}{ccc}
\mathcal{W}_{11}^{\prime}\left(\lambda^{*}\right) & 0 & 0 \\
0 & \mathcal{W}_{22}^{\prime}\left(\lambda^{*}\right) & 0 \\
0 & 0 & \mathcal{W}_{33}^{\prime}\left(\lambda^{*}\right)
\end{array}\right]
$$


where $\mathcal{W}_{11}^{\prime}\left(\lambda^{*}\right)=\beta_{12}+\beta_{02}-\lambda_{2}^{*}>0, \mathcal{W}_{22}^{\prime}\left(\lambda^{*}\right)=\beta_{12}+$ $\beta_{01}-\lambda_{1}^{*}-\frac{\beta_{12}^{2}}{\beta_{12}+\beta_{02}-\lambda_{2}^{*}}>0$, and $\mathcal{W}_{33}^{\prime}\left(\lambda^{*}\right)=\beta_{01}+\beta_{02}-\lambda_{0}^{*}-$ $\frac{\beta_{02}^{2}}{\beta_{12}+\beta_{02}-\lambda_{2}^{*}}-\frac{\mathbf{b}^{T} \mathbf{b}}{\beta_{12}+\beta_{01}-\lambda_{1}^{*}-\frac{\beta_{12}^{2}}{\beta_{12}+\beta_{02}-\lambda_{2}^{*}}}$, the zero eigenvalue of $\mathcal{W}\left(\lambda^{*}\right)$ (which is also the eigenvalue of $\bar{W}\left(\lambda^{*}\right)$ ) can only be

$$
\mathcal{W}_{33}^{\prime}\left(\lambda^{*}\right)=0 .
$$

Thus, we have that for two-step case, if $\mathbf{b} \neq 0$, the penalized matrix $\bar{W}\left(\lambda^{*}\right)$ has only one eigenvalue in 0 , by Lemma 3 , we have that the duality gap is zero, i.e., $d^{*}=f^{*}$.

On the other hand, consider the case when $\beta_{12}+\beta_{01}-\lambda_{1}^{*}-$ $\frac{\beta_{12}^{2}}{\beta_{12}+\beta_{02}-\lambda_{2}}=0$, From Lemma 4 , we have this requires that $\mathbf{b}=0$. Since the necessary and sufficient condition of

$$
\begin{aligned}
\mathbf{b} & =\left[\begin{array}{l}
\beta_{01} \cos \varphi_{010}+\frac{\beta_{02} \beta_{12}}{\beta_{12}+\beta_{02}-\lambda_{2}^{*}} \cos \left(\varphi_{020}-\varphi_{120}\right) \\
\beta_{01} \sin \varphi_{010}+\frac{\beta_{02} \beta_{12}}{\beta_{12}+\beta_{02}-\lambda_{2}^{*}} \sin \left(\varphi_{020}-\varphi_{120}\right)
\end{array}\right] \\
& =0
\end{aligned}
$$

is that both

$$
\varphi_{020}-\varphi_{120}=\varphi_{010}+(2 k+1) \pi, k \in\{-2,-1,0,1\}
$$

and

$$
\beta_{01}-\frac{\beta_{02} \beta_{12}}{\beta_{12}+\beta_{02}-\lambda_{2}^{*}}=0
$$

hold simultaneously. Thus, we have that if the following conditions hold simultaneously, $\bar{W}\left(\lambda^{*}\right)$ will have more than one zero eigenvalues.

$$
\begin{aligned}
& \varphi_{020}-\varphi_{010}-\varphi_{120}=(2 k+1) \pi, k \in\{-2,-1,0,1\} \\
& \beta_{01}-\frac{\beta_{02} \beta_{12}}{\beta_{12}+\beta_{02}-\lambda_{2}^{*}}=0 \\
& \beta_{12}+\beta_{01}-\lambda_{1}^{*}-\frac{\beta_{12}^{2}}{\beta_{12}+\beta_{02}-\lambda_{2}^{*}}=0
\end{aligned}
$$

which is (50), this completes the proof.

Remark 4: From Theorem 3, we have that one necessary condition to ensure that $\bar{W}\left(\lambda^{*}\right)$ has more than one zero eigenvalue is $\varphi_{020}-\varphi_{010}-\varphi_{120}=(2 k+1) \pi, k \in$ $\{-2,-1,0,1\}$. Since $\varphi_{i j 0} \in[-\pi, \pi)$, we have that $\varphi_{020}-$ $\varphi_{010}-\varphi_{120} \in[-3 \pi, 3 \pi)$, furthermore, since $\varphi_{120}, \varphi_{010}$ and $\varphi_{020}$ are all dependent on observation $\mathbf{z}_{k}^{i}$ and odometry $\mathbf{o}_{i}^{x y}$, let $\varphi=\varphi_{020}-\varphi_{010}-\varphi_{120}, \varphi$ can be seen as a continuous random variable in $[-3 \pi, 3 \pi)$. Let the probability density function of $\varphi$ be $f(\varphi)=\frac{1}{6 \pi}, \varphi \in[-3 \pi, 3 \pi)$, it is obvious that for an infinitesimal scalar $\epsilon$,

$$
\lim _{\epsilon \rightarrow 0} \int_{(2 k+1) \pi-\epsilon}^{(2 k+1) \pi+\epsilon} \frac{1}{6 \pi} d \varphi=\lim _{\epsilon \rightarrow 0} \frac{\epsilon}{3}=0
$$

which means that the probability when $\varphi=(2 k+1) \pi$ is zero, i.e., $\varphi_{020}-\varphi_{010}-\varphi_{120}=(2 k+1) \pi$ does not hold in practice.

Remark 5: It should be pointed out that by applying objective function (5), clear conditions for globally optimal solution to two-step point feature SLAM have been formulated in Theorem 3, this extends the results on solution to two-step SLAM without orientation part of odometry as proposed in (Wang et al. 2015) where objective function (3) is used. The following Example 2 shows the advantage of using objective function (5) instead of (3) for two-step case.

Example 2: Consider example of two-step with one feature case, the ground truth of pose $r_{1}, r_{2}$ are $\mathbf{x}_{r_{1}}=$ $\left[\begin{array}{ccc}5 & 0 & -3.1325\end{array}\right]^{T}, \mathbf{x}_{r_{2}}=\left[\begin{array}{lll}10 & 0 & -3.1403\end{array}\right]^{T}$, and the ground truth of the feature is $\left[\begin{array}{ll}10 & 5\end{array}\right]^{T}$, assume that the orientation part of odometry is affected by noises, and the observation to features and odometry data are $\mathbf{z}_{f}^{0}=\left[\begin{array}{ll}10 & 5\end{array}\right]^{T}, \mathbf{z}_{f}^{1}=\left[\begin{array}{ll}-5.0453 & -4.9543\end{array}\right]^{T}$, $\mathbf{z}_{f}^{2}=\left[\begin{array}{ll}-0.0065 & -5\end{array}\right]^{T}, \mathbf{o}_{1}^{x y}=\left[\begin{array}{ll}5 & 0\end{array}\right]^{T}, o_{1}^{\phi}=3.1363, \mathbf{o}_{2}^{x y}=$ $\left[\begin{array}{ll}-4.9998 & 0.0455\end{array}\right]^{T}, o_{2}^{\phi}=3.0935$. Using objective function (3), the two-step SLAM problem can be formulated as minimizing (29) of (Wang et al. 2015), which can be solved by using fminunc solver of Matlab, the result is that there are 2 local minima which depend on initial values, which are $\mathbf{x}=$ $\left[\begin{array}{llllllll}9.8877 & 4.8936 & 5.1123 & 0.1064 & 3.1065 & 10.6602 & -0.0210\end{array}\right.$ $-2.8721]^{T}$ and $\mathrm{x}=\left[\begin{array}{lllll}10.0960 & 5.0816 & 4.9040 & -0.0816\end{array}\right.$

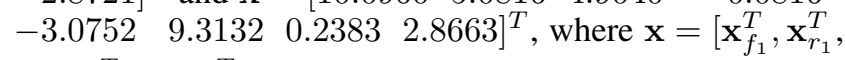
$\left.\phi_{r_{1}}, \mathbf{x}_{r_{2}}^{T}, \phi_{r_{2}}\right]^{T}$. However, using objective function (5), from observation and odometry data, we can verify that condition $\varphi_{020}-\varphi_{010}-\varphi_{120}=(2 k+1) \pi$ does not hold, from Theorem 3, we have that the solution to problem (48) is the globally optimal solution of the primal SLAM problem, i.e., $\mathbf{x}=\left[\begin{array}{lllll}9.9996 & 4.9970 & 5.0004 & 0.0030 & -\end{array}\right.$ $\left.\begin{array}{llll}3.1341 & 10.0095 & -0.0039 & -3.1365\end{array}\right]^{T}$.

\section{Simulation results to $m$-step SLAM}

In this section, some simulation results are presented to show the advantage of using the new objective function with Frobenius norm for $m$-step case of the SLAM problems.

For m-step ( $m \geq 3$ ) SLAM case, from Corollary 1, we have that the SLAM problem can be converted into minimizing the following objective function

$$
\begin{aligned}
& f_{0}\left(\phi_{r_{1}}, \cdots, \phi_{r_{m}}\right) \\
= & c_{1}-2 \sum_{0 \leq i<j \leq m} \beta_{i j} \cos \left(\phi_{r_{j}}-\phi_{r_{i}}-\varphi_{i j 0}\right)
\end{aligned}
$$

where parameters $c_{1}, \beta_{i j}, \varphi_{i j 0}$ are given in Corollary 1. However, if we use objective function (3), from (45) of (Wang et al. 2015), we have that the m-step SLAM problem will be converted into minimizing the following nonlinear 
function

$$
\begin{aligned}
& f_{0}\left(\phi_{r_{1}}, \cdots, \phi_{r_{m}}\right) \\
= & c_{0}-2 \sum_{0 \leq i<j \leq m} b_{i j} \cos \left(\phi_{r_{j}}-\phi_{r_{i}}-\phi_{i j 0}\right) \\
& +\sum_{i=1}^{m}\left(\operatorname{wrap}\left(o_{i}^{\phi}-\phi_{r_{i}}+\phi_{r_{i-1}}\right)\right)^{2} .
\end{aligned}
$$

Compare (54) with (55), it can be seen that (54) is simpler and $\operatorname{wrap}(\cdot)$ is not needed anymore. The following Example 3 shows that for some cases, minimizing objective function (54) may receive much better results than (55).

While we currently do not have theoretical results on the number of minima and global convergence for the general m-step case, we can obtain an a-posteriori optimality check as done in (Carlone et al. 2016) using Lemma 3. Similar to two-step case, the Lagrangian of primal optimization problem can be formulated as solving the following convex optimization problem:

$$
\begin{array}{r}
d^{*}=\max _{\lambda} \sum_{i=0}^{m} \lambda_{i} \\
\text { s.t., } \bar{W}(\lambda) \geq 0
\end{array}
$$

where $\bar{W}(\lambda)=W-\Lambda, \Lambda=\operatorname{diag}\left\{\lambda_{m} I_{2}, \lambda_{m-1} I_{2}, \ldots, \lambda_{0}\right\}$, with $W$ given in (31).

Through solving optimization problem $(56), \lambda^{*}$ is then obtained. Substitute $\lambda^{*}$ into $\bar{W}(\lambda)$, from Lemma 3 , we have that if $\bar{W}\left(\lambda^{*}\right)$ has one and only one zero eigenvalue, the duality gap between (56) and the primal optimization problem (27) is zero.

Example 3: Consider the following example, we let the ground truth of orientation part of robot poses be $-\pi+$ $0.05 *$ rand, where rand is a sample drawn from the uniform distribution in $[0,1]$, the orientation part of odometry is $\pi-$ $0.05 *$ rand, ground truth positions for both features and poses are shown in Fig. 2. In particular, for 4-step case, an example of pose ground truths are $\mathbf{x}_{r_{1}}=\left[\begin{array}{lll}5 & 0 & -3.1325\end{array}\right]^{T}$, $\mathbf{x}_{r_{2}}=\left[\begin{array}{lll}10 & 0 & -3.1403\end{array}\right]^{T}, \mathbf{x}_{r_{3}}=\left[\begin{array}{lll}15 & 0 & -3.1325\end{array}\right]^{T}, \mathbf{x}_{r_{4}}=$ $\left[\begin{array}{llll}20 & 0 & -3.1353\end{array}\right]^{T}$, and odometries are $\mathbf{o}_{1}=\left[\begin{array}{lll}5 & 0 & 3.1363\end{array}\right]^{T}$, $\mathbf{o}_{2}=\left[\begin{array}{lll}-5 & 0.0453 & 3.0935\end{array}\right]^{T}, \mathbf{o}_{3}=\left[\begin{array}{lll}-5 & 0.0063 & 3.1414\end{array}\right]^{T}$, $\mathbf{o}_{4}=\left[\begin{array}{lll}5 & 0.0457 & 3.1028\end{array}\right]^{T}$, Table 1 presents the results of percentage of convergence to global minima for minimizing objective function (54) and (55), where fminunc of Matlab is used to solve the minimization problem. From Table 1, it can be seen that using objective function (54) can receive much better convergence performance.

To demonstrate the advantage of objective function (54) further, consider the DLR dataset as presented in (Kurlbaum \& Frese 2009)(Zhao, Huang \& Dissanayake 2013). In order

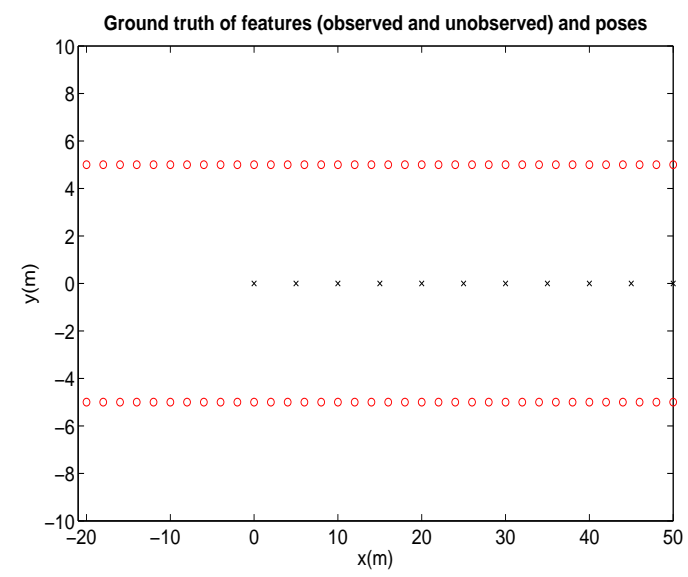

Fig. 2. Ground truth of features and poses. Black x-marks denote positions of robot poses, while red circles denote positions of features.

Table 1

Comparison of percentage of convergence to global minima (of 1000 simulations with arbitrary initial values) using fminunc).

\begin{tabular}{|c||c|c|c|}
\hline Number of robot poses $m$ & 4 & 10 & 20 \\
\hline Objective function (54) & $100 \%$ & $100 \%$ & $100 \%$ \\
\hline Objective function (55) & $34.9 \%$ & $23.3 \%$ & $15.5 \%$ \\
\hline
\end{tabular}

to compare the convergence of objective functions (54) and (55) with different levels of error and drift in the odometry, the orientation part of odometry is corrupted with different levels of additive zero mean Gaussian noise, the noise levels are represented by different variances of noises. In total four noise levels are tested to obtain statistical results in the following Example 4, i.e., the variances of noises are 0, 0.5, 1 and 2.

Example 4: In this example, we use the local map datasets available on OpenSLAM under project 2D-I-SLSJF, where 200 local maps are built from the DLR dataset, the results of different scenarios are obtained using fminunc in Matlab which are shown in Table 2 and Table 3, where it can be seen that the result using objective functions (54) receives better convergence performance. In addition, Fig. 3 shows the result when the orientation part of odometry is not corrupted by noise and the number of local maps is 20 , the objective function is 0.3717 at the optima $\mathrm{x}_{0}^{*}$, Table 4 shows the derivation between $x_{0}^{*}$ and the local minima $x_{1}^{*}$ when noise level is 1 , i.e., $\left\|\mathbf{x}_{0}^{*}-\mathbf{x}_{1}^{*}\right\|_{2}$, where it can be seen that there are two local minima corresponding to $f_{0}^{*}=16.1322$ and $f_{0}^{*}=21.2377$, respectively, obviously, the result corresponds to the objective function of 21.2377 is a local minimum. Furthermore, Fig. 4 shows the convergence of the cost vs the optimization step. 
Table 2

For DLR dataset, comparison of percentage of convergence to global minima (of 1000 simulations with arbitrary initial values) using fminunc for (54).

\begin{tabular}{|c||c|c|c|}
\hline Number of local maps & 4 & 10 & 20 \\
\hline Variance of noise: 0 & $100 \%$ & $100 \%$ & $100 \%$ \\
\hline Variance of noise: 0.5 & $100 \%$ & $100 \%$ & $100 \%$ \\
\hline Variance of noise: 1 & $100 \%$ & $100 \%$ & $100 \%$ \\
\hline Variance of noise: 2 & $100 \%$ & $100 \%$ & $100 \%$ \\
\hline
\end{tabular}

Table 3

For DLR dataset, comparison of percentage of convergence to global minima (of 1000 simulations with arbitrary initial values) using fminunc for (55).

\begin{tabular}{|c||c|c|c|}
\hline Total number of local maps & 4 & 10 & 20 \\
\hline Variance of noise : 0 & $100 \%$ & $100 \%$ & $100 \%$ \\
\hline Variance of noise: 0.5 & $100 \%$ & $100 \%$ & $100 \%$ \\
\hline Variance of noise: 1 & $100 \%$ & $74.4 \%$ & $72.5 \%$ \\
\hline Variance of noise: 2 & $100 \%$ & $50.1 \%$ & $33.5 \%$ \\
\hline
\end{tabular}

Table 4

Deviation of local minima

\begin{tabular}{|c||c|c|}
\hline objective function $f_{0}^{*}$ & 16.1322 & 21.2377 \\
\hline Deviation & 17.66 & 18.38 \\
\hline
\end{tabular}

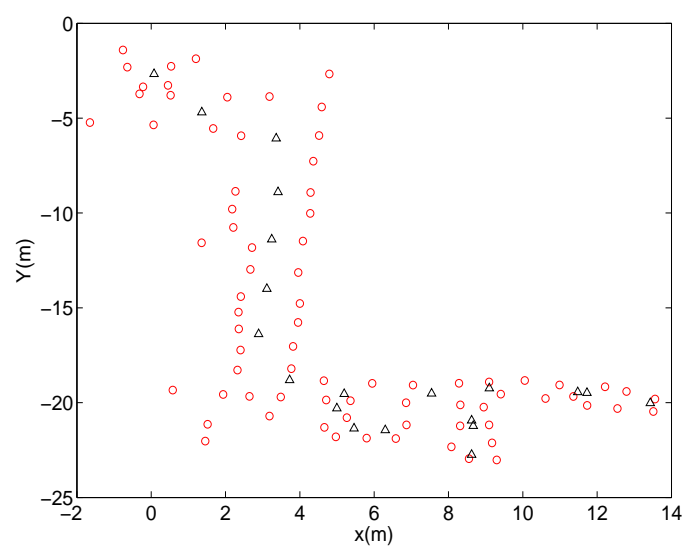

Fig. 3. Part of DLR dataset. Black triangles denote positions of robot poses, while red circles denote positions of features.

Example 5: Consider 10-step case, ground truths of positions of features and poses are chosen as shown in Fig. 2, and orientation of poses are arbitrarily chosen. Observation of features and odometries are set to be ground truth plus different levels of noises with variances being $0.1,1,3,5$ and 10. For each level of noise, through solving optimization problem (56), we substitute its solution $\lambda^{*}$ into $\bar{W}(\lambda)$ and see whether SZEP condition holds, Table 5 shows average numbers of cases when SZEP condition holds out of 1000 simulation for each level of noises, which is consistent

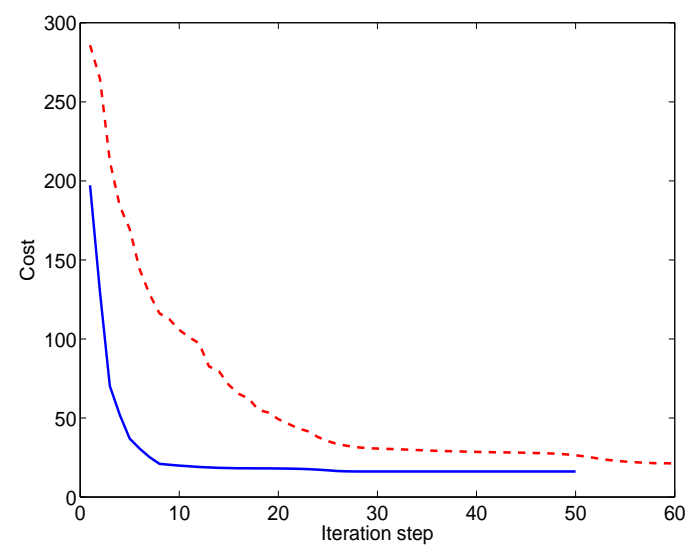

Fig. 4. Convergence of the cost vs optimization step, where the blue line corresponds to the cost $f_{0}^{*}=16.1322$ at optima, and the red dash line corresponds to the cost $f_{0}^{*}=21.2377$ at optima.

Table 5

Average number when SZEP condition is satisfied (of 1000 simulations) for different noise levels.

\begin{tabular}{|c||c|c|c|c|c|}
\hline Variance of noise & 0.1 & 1 & 3 & 5 & 10 \\
\hline SZEP condition holds & 1000 & 1000 & 986 & 964 & 948 \\
\hline
\end{tabular}

with the numerical analysis for the pose-based SLAM case in Figure 4 of (Carlone et al. 2016).

\section{Conclusions}

In this paper, the advantage of introducing Frobenius norm into objective function in SLAM has been demonstrated using both theoretical analysis and numerical examples. It has been proved that the strong duality always holds for one-step point feature SLAM with spherical covariance, and for twostep case, the strong duality also holds as long as three strict conditions are not satisfied simultaneously. It is also shown that for arbitrary m-step point feature SLAM problem, if SZEP condition is satisfied, the globally optimal solution to primal SLAM problem can be obtained by solving a convex optimization problem. Simulation examples have verified the conclusions proposed in this paper. In the future, the following points will be under our further research directions. Firstly, it is necessary to analyze the effect of choosing local optimum as solution, especially for real world SLAM problems, and the effect on the ability to close loops. Secondly, since practical SLAM implementations use non-linear leastsquares solvers, it is useful to apply the convergence results proposed in this paper to those other optimization schemes. Thirdly, note that angle-axis parameterizations and rotation matrices are related via the exponential map for $\mathrm{SO}(3)$, it is important to further investigate the extension to $3 \mathrm{D}$ case and a version of objective function (5) that directly works on quaternion or angle-axis parameterizations. 


\section{References}

Cadena, C., Carlone, L., Carrillo, H., Latif, Y., Scaramuzza, D., Neira, J., Reid, I. \& Leonard, J. (2016). Past, present, and future of simultaneous localization and mapping: toward the robust-perception age, IEEE Transactions on Robotics 32(6), 1309-1332.

Carlone, L. (2013). A convergence analysis of pose graph optimization via gauss-newton methods, Proceedings of the IEEE International Conference on Robotics and Automation (ICRA), Karlsruhe, Germany, pp. 965-972.

Carlone, L., Aragues, R., Castellanos, J. A. \& Bona, B. (2014). A fast and accurate approximation for planar pose graph optimization, International Journal of Robotics Research 33(7), 965-987.

Carlone, L., Calafiore, G., Tommolillo, C. \& Dellaert, F. (2016). Planar pose graph optimization: Duality, optimal solutions, and verification, IEEE Transactions on Robotics 32(3), 545-565.

Carlone, L. \& Dellaert, F. (2015). Duality-based verification techniques for $2 \mathrm{~d}$ slam, In Proc. IEEE Int. Conf. Robot. Autom., Seattle, WA, USA, pp. 4589-4596.

Carlone, L., Tron, R., Daniilidis, K. \& Dellaert, F. (2015). Initialization techniques for $3 \mathrm{~d}$ slam: a survey on rotation estimation and its use in pose graph optimization, In Proc. IEEE Int. Conf. Robot. Autom., Seattle, WA, USA, pp. 4597-4604.

Dellaert, F. \& Kaess, M. (2006). Square Root SAM: Simultaneous localization and mapping via square root information smoothing, Intl. J. of Robotics Research 25(12), 1181-1204.

Gallier, J. (2010). The schur complement and symmetric positive semidefinite (and definite) matrices, available online: http://www.cis.upenn.edu/ jean/schur-comp.pdf.

Grisetti, G., Stachniss, C. \& Burgard, W. (2009). Non-linear constraint network optimization for efficient map learning, IEEE Transactions on Intelligent Transportation Systems 10(3), 428-439.

Grisetti, G., Stachniss, C., Grzonka, S. \& Burgard, W. (2007). A tree parameterization for efficiently computing maximum likelihood maps using gradient descent, Proceedings of Robotics: Science and Systems, Atlanta, GA, USA.

Gutmann, J. S. \& Konolige, K. (1999). Incremental mapping of large cyclic environments, In Proc. IEEE Int. Symp. Comput. Intell. Robot. Autom., Monterey, CA, USA, pp. 318-325.

Hartley, R., Trumpf, J., Dai, Y. \& Li, H. (2013). Rotation averaging, International Journal of Computer Vision 103(3), 267-305.

Huang, S., Lai, Y., Frese, U. \& Dissanayake, G. (2010). How far is slam from a linear least squares problem?, IEEE/RSJ International Conference on Intelligent Robots and Systems (IROS), Taipei, Taiwan, pp. 3011-3016.
Kaess, M., Johannsson, H., Roberts, R., Ila, V., Leonard, J. \& Dellaert, F. (2012). isam2: Incremental smoothing and mapping using the bayes tree, International Journal of Robotics Research 31, 217-236.

Khosoussi, K., Huang, S. \& Dissanayake, G. (2015). Exploiting the separable structure of slam, 2015 Robotics: Science and Systems Conference (RSS), Massachusetts, USA.

Kurlbaum, J. \& Frese, U. (2009). A benchmark data set for data association, Technical Report 017-02/2009,SFB TR/8.

Liu, M., Huang, S., Dissanayake, G. \& H.Wang (2012). A convex optimization based approach for pose slam problems, In Proc. IEEE/RSJ Int. Conf. Intell. Robots Syst., Vilamoura, Portugal, pp. 1898-1903.

Lu, F. \& Milios, E. (1997). Globally consistent range scan alignment for environment mapping, Autonomous Robots 4(4), 333-349.

Olson, E., Leonard, J. J. \& Teller, S. (2006). Fast iterative optimization of pose graphs with poor initial estimates, IEEE International Conference on Robotics and Automation (ICRA), Orlando, Florida, pp. 2262-2269.

Rosen, D., Carlone, L., Bandeira, A. \& Leonard, J. (2016). Sesync: A certifiably correct algorithm for synchronization over the special euclidean group, Workshop on the Algorithmic Foundations of Robotics (WAFR), San Francisco, CA.

Rosen, D. M., DuHadway, C. \& Leonard, J. J. (2015). A convex relaxation for approximate global optimization in simultaneous localization and mapping, In Proc. IEEE Int. Conf. Robot. Autom., Seattle, WA, USA, pp. 5822-5829.

Thrun, S. \& Montemerlo, M. (2006). The graphslam algorithm with application to large-scale mapping of urban sturcture, International Journal of Robotics Research 25(5-6), 403-429.

Wang, H., Hu, G., Huang, S. \& Dissanayake, G. (2012). On the structure of nonlinearities in pose graph slam, 2012 Robotics: Science and Systems Conference (RSS), Sydney, Australia.

Wang, H., Huang, S., Frese, U. \& Dissanayake, G. (2013). The nonlinearity structure of point feature slam problems with spherical covariance matrices, Automatica 49(10), 3112-3119.

Wang, H., Huang, S., Khosoussi, K., Frese, U., Dissanayake, G. \& Liu, B. (2015). Dimensionality reduction for point feature slam problems with spherical covariance matrices, Automatica 51(1), 149-157.

Zhao, L., Huang, S. D. \& Dissanayake, G. (2013). Linear slam: A linear solution to the feature-based and pose graph slam based on submap joining, IEEE/RSJ International Conference on Intelligent Robots and Systems (IROS), Tokyo, Japan, pp. 24-30. 